Disclosure Information N. Prokopi: None. M. Heeren: None. I. Milenova: None. K. van Pul: None. T. Muijlwijk: None. M. Arends: None. S. van der Velde: None. K. Vrijland: None. A. van Weverwijk: None. K. de Visser: None. R. van de Ven: None. T. de Gruijl: None.

\section{P09.03 CATHEPSIN S ALTERATIONS INDUCE A TUMOR- PROMOTING IMMUNE MICROENVIRONMENT IN FOLLICULAR LYMPHOMA}

\begin{abstract}
${ }^{1} \mathrm{JA}$ Hildebrand*, ${ }^{1} \mathrm{D}$ Bararia, ${ }^{1} \mathrm{~S}$ Stolz, ${ }^{1} \mathrm{~S}$ Häbe, ${ }^{2} \mathrm{~F}$ Osorio-Barrios, ${ }^{3} \mathrm{MD}$ Bartoschek, ${ }^{1} \mathrm{E}$ Gaitzsch, ${ }^{1} \mathrm{~V}$ Jurinovic, ${ }^{4} \mathrm{~K}$ Rautter, ${ }^{5} \mathrm{C}$ Ludwig, ${ }^{3} \mathrm{~S}$ Bultmann, ${ }^{3} \mathrm{H}$ Leonhardt, ${ }^{6} \mathrm{~S}$ Eustermann, ${ }^{6} \mathrm{~K}$ Hopfner, ${ }^{7} \mathrm{~W}$ Hiddemann, ${ }^{7} \mathrm{M}$ Bergwelt, ${ }^{8} \mathrm{M}$ Schmidt-Supprian, ${ }^{9} \mathrm{MB}$ Sárosi, ${ }^{10} \mathrm{M}$ Rudelius, ${ }^{1} \mathrm{~V}$ Passerini, ${ }^{11} \mathrm{~J}$ Mautner, ${ }^{1} \mathrm{O}$ Weigert. ${ }^{1}$ Experimental leukemia and lymphoma research, LMU, university clinic, Munich, Germany; ${ }^{2}$ Hematology and Oncology, TUM, Klinikum rechts der Isar, Munich, Germany; ${ }^{3}$ Department of Biology II, LMU, Center for Integrated Protein Science Munich (CIPSM), Munich, Germany; ${ }^{4}$ Immunoanalytics: Research Group Tissue Control of Immunocytes and Core Facility, Helmholtz Zentrum, Munich, Germany; ${ }^{5}$ Bavarian Center for Biomolecular Mass Spectrometry (BayBioMS), TUM, Munich, Germany; ${ }^{6}$ Gene Center, LMU, Munich, Germany; ${ }^{7}$ Department of Internal Medicine III, LMU, Munich, Germany; ${ }^{8}$ Hematology and Oncology, Klinikum rechts der Isar, TUM, Munich, Germany; Institute of Inorganic Chemistry, Faculty of Chemistry and Mineralogy, Leipzig, Germany; ${ }^{10}$ Institute of Pathology, LMU, Munich, Germany; ${ }^{11}$ Helmholtz Zentrum, Munich, Germany
\end{abstract}

\subsection{6/jitc-2020-ITOC7.103}

Background By targeted DNA sequencing of 305 diagnostic follicular lymphoma (FL) biopsies, we identified somatic mutations of Cathepsin S (CTSS) in 8\% of cases (24/305), mostly clustered at Y132 (19/24) converting Y to D (16/19). Another $13 \%$ of FL had CTSS amplifications (37/286), associated with higher CTSS expression $(P=0.05)$. CTSS is a cysteine protease that is highly expressed in endolysosomes of antigen presenting cells and malignant B-cells. CTSS is involved in proteolytical processing of antigenic peptides for presentation on MHCII to be recognized by antigen specific CD4 ${ }^{+}$T-cells. $^{1}$ CTSS is synthesized as an inactive zymogen, which is converted to its active form by autocatalytic cleavage of the autoinhibitory propeptide (pro-CTSS).

Materials and Methods We used CRISPR/Cas9 to introduce CTSS Y132D into Karpas422, a B-cell lymphoma cell line that harbors the FL hallmark translocation $\mathrm{t}(14 ; 18)$. We purified pro-CTSS WT and Y132D and assayed the in vitro autocatalytic cleavage over time. We then tested the impact of CTSS on $\mathrm{CD}^{+}{ }^{+} \mathrm{T}$-cell activation in co-culture assays, in a previously described in vivo model ${ }^{2}$ which we slightly modified to reflect FL-like conditions, and in primary patient samples.

Results Single-cell derived Y132D mutant Karpas422 clones showed $>3$-fold higher ratios of active CTSS to pro-CTSS $(\mathrm{N}=4, P=0.0003)$. Immunoprecipitated CTSS Y132D had $>3$-fold higher in vitro substrate cleavage activity compared to CTSS wild type (WT) $(\mathrm{N}=6, P=0.001)$ which was mediated by an accelerated conversion from pro-CTSS to active CTSS (11 minutes for CTSS Y132D vs 17 minutes for CTSS WT; $\mathrm{N}=3, P=0.04)$. Molecular dynamics simulations showed that the Y132D mutation shortens the distances by $\sim 2 \AA$ between the catalytic triad of active CTSS (C139, H278, N298) and a stretch of amino acids from the proform (L80, G81, D82, S94), which could facilitate intramolecular cleavage. The higher substrate cleavage activity of CTSS Y132D came along with a high capacity to stimulate antigen specific $\mathrm{CD}^{+} \mathrm{T}$ cell responses in vitro and in vivo. Additionally, CTSS overexpression could phenocopy this high $\mathrm{CD}^{+} \mathrm{T}$ cell activation. Lastly, we aimed to correlate CTSS aberrations with clinical outcome in patients who received standard immunochemotherapy (R-CHOP) for advanced FL ( $=51$ with available CTSS mutation and gene expression data). Compared to all other patients $(\mathrm{N}=34)$, patients with CTSS Y132 mutations or CTSS overexpression $(\mathrm{N}=17)$ had longer failure free survival $(P=0.012)$.

Conclusions Here, we provide biochemical, structural, functional and clinical evidence that aberrant CTSS activity induces a supportive immune microenvironment in FL. We propose that aberrant CTSS activity can elicit a CD4 ${ }^{+}$T-cell driven tumor-promoting immune response, which could be amplified within the microenvironment and substantially impact the biology and clinical course of the disease. Thus, aberrant CTSS activity is a promising biomarker and therapeutic target in FL and potentially also other tumors.

\section{REFERENCES}

1. Riese, R.J., et al., Essential role for cathepsin $S$ in $M H C$ class II-associated invariant chain processing and peptide loading. Immunity 1996; 4(4): p. 357-66.

2. Kim, K.J., et al., Establishment and characterization of BALB/C lymphoma lines with B cell properties. J Immunol 1979; 122(2): p. 549-54

Disclosure Information J.A. Hildebrand: None. D. Bararia: None. S. Stolz: None. S. Häbe: None. F. Osorio-Barrios: None. M.D. Bartoschek: None. E. Gaitzsch: None. V. Jurinovic: None. K. Rautter: None. C. Ludwig: None. S. Bultmann: None. H. Leonhardt: None. S. Eustermann: None. K. Hopfner: None. W. Hiddemann: None. M. Bergwelt: None. M. Schmidt-Supprian: None. M.B. Sárosi: None. M. Rudelius: None. V. Passerini: None. J. Mautner: None. O. Weigert: None.

\section{P09.04 ONCOLYTIC H5N1 INFLUENZA STRAIN DISPLAYS SUPERIOR THERAPEUTIC PROPERTIES INDEPENDENT OF IMMUNO-STIMULATORY INTERLEUKIN-2 TRANSGENE EXPRESSION}

'J Kabiljo*, 'I Kuznetsova, '1 Homola, 'S Prodinger, ${ }^{1,2}$ J Laengle, 'M Sachet, ${ }^{3} \mathrm{~A}$ Egorov, ${ }^{1,2} \mathrm{M}$ Bergmann. 'Division of General Surgery, Department of Surgery, Comprehensive Cancer Center Vienna, Medical University of Vienna, Waehringer Guertel 18-20, A-1090 Vienna, Austria, Vienna, Austria; ${ }^{2}$ Ludwig Boltzmann Institute Applied Diagnostics, Medical University of Vienna, Waehringer Guertel 18-20, 1090 Vienna, Austria, Vienna, Austria; ${ }^{3}$ Research Institute of Influenza, Russian Academy of Medical Sciences, Prof. Popova Str. 15/ 17196376 St. Petersburg, Russia., St. Petersburg, Russian Federation

\subsection{6/jitc-2020-ITOC7.104}

Background Oncolytic viruses are becoming an integral part of immunological approaches to cancer treatment. Interleukin-2 (IL-2) is known to stimulate cytotoxic T-cells, and might therefore be a reasonable cargo to enhance the therapeutic effect of such viruses. However, IL-2 is also known to promote immunosuppressive regulatory T-cells (T-reg). We investigated the impact of virally expressed IL-2 on induction of regulatory T-cells. We further investigated the effect of virally expressed IL-2 on the therapeutic efficacy of influenza A H1 and H5 subtypes.

Materials and Methods Survival of B16 melanoma xenograft bearing mice upon treatment with various oncolytic influenza viruses was examined. Effect of these viruses on PBMC gathered from 4 young healthy volunteers and murine and human melanoma cell lines was examined utilizing multiple flow cytometry protocols.

Results Viral IL-2 expression did not alter viral growth and was stable up to multiple passages in cell cultures. In human PBMC viral expression of IL-2 did not enhance differentiation 
of T-cells into a regulatory phenotype. In a murine B16 xenograft model IL-2 expression significantly enhanced therapeutic effects of an $\mathrm{H} 1$ oncolytic influenza virus. Expressed within the background of H5 hemagglutinin, IL-2 did not lead to a significant enhancement of therapeutic efficacy. Interestingly, the empty influenza H5 subtype was significantly more potent in treating B16 xenograft tumors than the H1 subtype, regardless of IL-2 expression. In primary human PBMC models, the virus based on $\mathrm{H} 1$ hemagglutinin led to enhanced CD8 T-cell activation compared to H5. This effect was further enhanced by IL-2 expression, although all viruses led to significant activation. Surprisingly, viruses based on H1 hemagglutinin led to increased expression of the immune checkpoint PD-1. The virus based on H5 hemagglutinin did not lead to upregulation of PD-1, indicating a favorable balance between activation and exhaustion. Infection with the $\mathrm{H} 5$ based virus led to both enhanced apoptosis and immunogenic calreticulin exposure in human and murine melanoma cell lines compared to $\mathrm{H} 1$.

Conclusions IL-2 does not promote T-regs, when expressed in a viral background. $\mathrm{H} 1$ viruses induced $\mathrm{PD}-1$ more potently than $\mathrm{H} 5$ viruses. The choice of viral entry protein is more relevant for the therapeutic effect of the virus, than the expression of a T-cell stimulating cytokine such as IL-2. Efficacy of oncolytic viral treatment appears to depend more on viral growth than on virally expressed T-cell promoting cargo. Disclosure Information J. Kabiljo: None. I. Kuznetsova: None. J. Homola: None. S. Prodinger: None. J. Laengle: None. M. Sachet: None. A. Egorov: A. Employment (full or part-time); Modest; Vacthera Bio Tech GmbH. M. Bergmann: A. Employment (full or part-time); Modest; Vacthera Bio Tech GmbH.

\section{P09.05 IMMUNOGENICITY INDUCED BY THE ACADEMIC CHIMERIC ANTIGEN RECEPTOR CAR19 (ARI-0001) IN PATIENTS WITH CD19-POSITIVE RELAPSED/REFRACTORY B-CELL MALIGNANCIES RECRUITED INTO THE CART19- BE-01 CLINICAL TRIAL}

\begin{abstract}
1,2N Klein-González* ${ }^{*}$ 1,2EA González-Navarro, 1,2 A Bartoló-Ibars, ${ }^{3} \mathrm{~V}$ Ortiz-Maldonado, ${ }^{4} \mathrm{M}$ Torrebadell, $5,6 \mathrm{M}$ Castellà, ${ }^{1,2} \mathrm{D}$ Benítez, 1,2M Caballero-Baños, 1,2R Cabezón, 1,2 M Español, ${ }^{3} \mathrm{~T}$ Baumann, ${ }^{3} \mathrm{E}$ Giné, ${ }^{7,8} \mathrm{P}$ Castro, ${ }^{3,5,8}$ J Esteve, ${ }^{2,5,8}$ J Yagüe, ${ }^{4} \mathrm{~S}$ Rives, ${ }^{3,5,8} \AA_{\mathrm{A}}$ Álvaro Urbano-Ispizua, ${ }^{3,5}$ J Delgado, $1,2,5,8 \mathrm{M}$ Juan. ${ }^{1}$ Immunotherapy Unit, HSJD-Hospital Clínic Barcelona, Barcelona, Spain; ${ }^{2} / m m u n o l o g y, ~ C D B$, Hospital Clínic Barcelona, Barcelona, Spain; ${ }^{3}$ Hematology, ICMHO, Hospital Clínic Barcelona, Barcelona, Spain; ${ }^{4}$ Hematology, Hospital Sant Joan de Déu (HSJD), Barcelona, Spain; Institut d'Investigacions Biomèdiques August Pi i Sunyer - IDIBAPS, Barcelona, Spain; ${ }^{6}$ Banc de Sang i Teixits (BST), Barcelona, Spain; ${ }^{7}$ Medical Intensive Care Unit, Hospital Clínic Barcelona, Barcelona, Spain; ${ }^{8}$ University of Barcelona, Barcelona, Spain
\end{abstract}

\subsection{6/jitc-2020-ITOC7.105}

Background Chimeric Antigen Receptor (CAR)-T cells directed against CD19 have induced high rates of response in patients with relapsed/refractory (R/R) B-cell malignancies. Two CD19targeting constructs have been approved by the FDA and EMA (Yescarta ${ }^{\circledR}$, Kymriah ${ }^{\circledR}$ ) for B lymphoblastic leukemia (B-ALL) and aggressive lymphoma. Despite deep remissions, there are still major challenges and disparate data are reported about the immunogenicity induced by CART-cell therapy. On May/2017, the Spanish Agency of Medicines approved our first clinical trial (clinicaltrials.gov NCT03144583) with a fully academic CART-19.

Materials and Methods Eligibility criteria included R/R B-ALL (adult and pediatric), non-Hodgkin's lymphoma (NHL) and chronic lymphocytic leukemia(CLL) who failed standard therapy. The primary objective of the study was safety and secondary objectives were response rate and its duration. The humoral anti-CART response was assessed by a (cell-based) fluorescence assay to detect human anti-murine antibodies (HAMA) in patients sera. Assessment was conducted at different time points: 1) at baseline (pre-dose), 2) on day 14 after the administration of ARI-0001 cells, 3) on day 28, 4) on day 100, and 5) every 3 months thereafter.

Results Forty-seven patients (37 adults/10 pediatrics) received ARI-0001 cells. Thirty-eight patients had a diagnosis of $\mathrm{R} / \mathrm{R}$ B-ALL (28 adults and 10 children); all but 5 had relapsed after allogeneic hematopoietic stem cell transplant (HCT). Seven patients had a diagnosis of NHL, four of them $(57 \%)$ had relapsed after HCT, and 2 patients had a diagnosis of CLL (2). Median age was 27 years (3-68). After conditioning with fludarabine $(90 \mathrm{mg} / \mathrm{m} 2)$ and cyclophosphamide $(900$ $\mathrm{mg} / \mathrm{m} 2$ ), a total dose of $0.5-5 \times 10^{6}$ ARI-0001 cells $/ \mathrm{kg}$ was infused. Autologous T-cells from peripheral blood were expanded and transduced with a lentivirus to express a CAR with a single-chain variable fragment $(\mathrm{scFv})$ with anti-CD19 specificity, conjugated with the co-stimulatory regions $4-1 \mathrm{BB}$ and $\mathrm{CD} 3 \mathrm{z}$. The $\mathrm{scFv}$ was originated from a mouse monoclonal antibody A3B1. Twenty-five per cent of the patients tested positive for the presence of anti-CAR antibodies, all of them post-dose, in contrast to previous data reported on Kymriah $^{\circledR}$ with a significant presence of pre-dose anti-murine CAR19 antibody. Of these 12 patients, 8 patients presented with a weak, and 4 patients with a strong presence of HAMA. The last 4 patients had lost the effectiveness of the CART- therapy at that time point, reflected by simultaneous B-cell recovery in the periphery. Moreover, three of them received a second dose of CART-19, which did not revert the relapse.

Conclusions To conclude, these data suggest the importance of the immunogenicity induced by CART-cell therapies. Immune monitoring should include the assessment of humoral response, especially before considering a second dose after relapse.

Disclosure Information N. Klein-González: None. E.A. González-Navarro: None. A. Bartoló-Ibars: None. V. Ortiz-Maldonado: None. M. Torrebadell: None. M. Castellà: None. D. Benítez: None. M. Caballero-Baños: None. R. Cabezón: None. M. Español: None. T. Baumann: None. E. Giné: None. P. Castro: None. J. Esteve: None. J. Yagüe: None. S. Rives: None. Á. Álvaro Urbano-Ispizua: None. J. Delgado: None. M. Juan: None.

\section{P09.06 'AN ENHANCED CRISPR TOOL FOR TREATING CHRONIC MYELOGENOUS LEUKEMIA'}

${ }^{1} \mathrm{D}$ Lainšček ${ }^{*},{ }^{1} \mathrm{~V}$ Forstnerič, ${ }^{1} \mathrm{~S}$ Malenšek, ${ }^{1} \mathrm{M}$ Skrbinek, ${ }^{2} \mathrm{M}$ Sever, ${ }^{1,3} \mathrm{R}$ Jerala. ${ }^{1}$ National institute of Chemistry, Ljubljana, Slovenia; ${ }^{2}$ University medical centre Ljubljana, Ljubljana, Slovenia; ${ }^{3}$ EN-FIST Centre of Excellence, Ljubljana, Slovenia

\subsection{6/jitc-2020-ITOC7.106}

Background Chronic myeloid leukemia (CML) is a myeloproliferative neoplastic disease, occurring in 1 to 2 cases per 100.000 adults, which accounts this type of cancer for approximately $15 \%$ of newly diagnosed leukemia in adult patients. The diagnosis is based upon the genetic translocation between the $\mathrm{t}(9 ; 22)(\mathrm{q} 34 ; \mathrm{q} 11.2)$, resulting in formation 